\author{
B. Trzeciak - C. Da Silva - E. G. Ferreiro - C. Hadjidakis • \\ D. Kikola - J. P. Lansberg 1 L. Massacrier - J. Seixas • \\ A. Uras · Z. Yang
}

\title{
Heavy-Ion Physics at a Fixed-Target Experiment Using the LHC Proton and Lead Beams (AFTER@LHC): Feasibility Studies for Quarkonium and Drell-Yan Production
}

Received: 10 March 2017 / Accepted: 7 June 2017 / Published online: 7 July 2017

(C) The Author(s) 2017. This article is an open access publication

\begin{abstract}
We outline the case for heavy-ion-physics studies using the multi-TeV lead LHC beams in the fixed-target mode. After a brief contextual reminder, we detail the possible contributions of AFTER@LHC to heavy-ion physics with a specific emphasis on quarkonia. We then present performance simulations for a selection of observables. These show that $\Upsilon(n S), J / \psi$ and $\psi(2 S)$ production in heavy-ion collisions can be studied in new energy and rapidity domains with the LHCb and ALICE detectors. We also discuss the relevance to analyse the Drell-Yan pair production in asymmetric nucleus-nucleus collisions to study the factorisation of the nuclear modification of partonic densities and of further quarkonium states to restore their status of golden probes of the quark-gluon plasma formation.
\end{abstract}

This article belongs to the Topical Collection "New Observables in Quarkonium Production".

B. Trzeciak $(\bowtie)$

Institute for Subatomic Physics, Utrecht University, Utrecht, The Netherlands

E-mail: b.a.trzeciak@uu.nl

C. Da Silva

P-25, Los Alamos National Laboratory, Los Alamos, NM 87545, USA

E. G. Ferreiro

Departamento de Física de Partículas, Universidade de Santiago de Compostela, 15782 Santiago de Compostela, Spain

C. Hadjidakis - J. P. Lansberg · L. Massacrier

IPNO, CNRS-IN2P3, Univ. Paris-Sud, Université Paris-Saclay, Orsay, France

D. Kikola

Faculty of Physics, Warsaw University of Technology, Warsaw, Poland

J. Seixas

LIP and IST, Lisbon, Portugal

A. Uras

IPNL, Lyon, France

Z. Yang

Tsinghua University, Beijing, China 


\section{Contents}

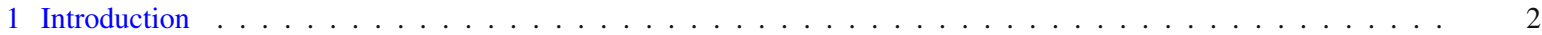

2 Quark-Gluon-Plasma Studies with AFTER@LHC . . . . . . . . . . . . . . . . . . . . . . . . . 3

3 Simulations . . . . . . . . . . . . . . . . . . . . . . . . . . . . . .

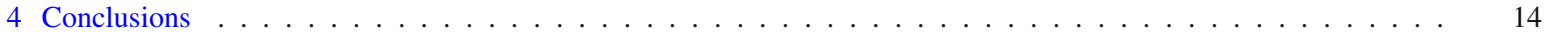

\section{Introduction}

Hadro-production experiments in the fixed-target mode have indisputably played a fundamental role in the history of quarkonium physics. This began with the co-discovery of the $J / \psi$ [1] in 1974 at the Brookhaven National Laboratory and followed by the discovery of the $\Upsilon$ [2] and the first observation of $h_{c}$ [3] at the Fermi National Laboratory. The dedicated heavy-ion program at the Super Proton Synchrotron (SPS) at CERN then uncovered many novel and unexpected features of quark and gluon dynamics, including the anomalous suppression of $J / \psi[4]$ in $\mathrm{PbPb}$ collisions, and helped discover the strong non-factorising nuclear suppression of the $J / \psi$ hadro-production at high $x_{F}$ [5]. Let us recall that the first observation of $J / \psi$-pair production was carried out at the SPS as early as in 1982 [6]. Fixed-target experiments have also played a central role in measuring Drell-Yan pair production, in particular below the bottomonium region [7-10].

In this context, we find it important to remind that collisions of the proton and heavy-ion LHC beams on fixed targets open a remarkably large range of physics opportunities [11-46]. The AFTER@LHC project bears on them and aims at an ambitious heavy-ion, spin and hadron-structure physics programme. These opportunities result from a high luminosity - be it with an internal gas target or with an extracted beam- together with a relatively high center-of-mass system (c.m.s.) energy of $115 \mathrm{GeV}$ per nucleon with a $7 \mathrm{TeV}$ proton beam and $72 \mathrm{GeV}$ per nucleon with a $2.76 \mathrm{~A}$.TeV lead beam. Besides, the boost typical of the fixed-target mode makes forward detectors such as LHCb and the ALICE muon spectrometer nearly ideal to probe the negative $x_{F}$ region, which is essentially uncharted despite its interest. Also, mid-rapidity detectors such as the central barrel of ALICE allow to access the far-backward region down to the target fragmentation limit.

The AFTER@LHC-energy range stands half way between the c.m.s. energies of SPS and the Relativistic Heavy Ion Collider (RHIC), allowing, in particular, for detailed studies of the bottomonium production and dynamics and offering a very large physical acceptance for detectors covering between 1 and 3 units of rapidity.

In $[28,47]$, we have focused on the possibility offered by the LHC nine-months-per-year proton program during which AFTER @LHC would be able to study the production of quarkonia in $p p, p d$ and $p A$ collisions with an unparalleled statistical accuracy down to $x_{F} \rightarrow-1$. Such high-precision quarkonium-production measurements in $p p$ are essential [48-50] to solve longstanding puzzles in $J / \psi$ and $\Upsilon$ production and for the understanding of their behavior in the (Cold) Nuclear Matter (CNM).

AFTER@LHC can also provide extremely relevant information on the Quark-Gluon Plasma (QGP), which should be created at $\sqrt{s_{N N}}=72 \mathrm{GeV}$ in $\mathrm{Pb} A$ collisions. Despite the decrease in the c.m.s. energy, the total number of produced quarkonia similar to that of LHC at $\sqrt{s_{N N}}=2.76$ and $5.5 \mathrm{TeV}$ and RHIC at $\sqrt{s_{N N}}=200 \mathrm{GeV}$ is to be expected. These are two orders of magnitude larger than the ones obtained at RHIC at $\sqrt{s_{N N}}=62 \mathrm{GeV}$. The first results from the LHC at $\sqrt{s_{N N}}=2.76$ and $5.02 \mathrm{TeV}$ [51] confirmed that the pattern of quarkonium (anomalous) suppression at high energy is very intricate with subtle $y$ and $p_{T}$ dependencies. Low energy experiments, where the recombination process [51] is not expected to be significant, can then play a key role in understanding the underlaying physics processes. Such a role will become central if it is possible to carry out measurements of $\chi_{c}$ and even $\chi_{b}$ production and suppression in heavy-ion collisions-2 measurements thus far not performed in any other experimental configuration. The quest for the sequential suppression of quarkonia as a QGP thermometer would then become realistic again.

In addition, the measurement of the Drell-Yan-pair production in asymmetric nucleus-nucleus collisions provides a unique opportunity to test the factorisation of the initial-state nuclear effect such as the nuclear PDFs. Such a factorisation is routinely assumed at high energies but a number of non-linear effects can violate it. If these are significant, the nuclear effects from both colliding nucleus do not add up linearly and this would simply prevent us to use the information gained from proton-nucleus or electron-nucleus collisions to characterise the initial stage of nucleus-nucleus collisions. Because of a very high background at the LHC in the collider mode, such measurements are not even feasible in $\mathrm{PbPb}$ collisions. At the LHC, the studies of asymmetric nucleus-nucleus systems are not planned, and such a measurement is very difficult at RHIC. With the lower energy at AFTER@LHC, the studies may be within reach if sufficient different systems are used $(p \mathrm{~Pb}, \mathrm{~Pb} p, \mathrm{PbXe}, p \mathrm{Xe}, \ldots)$ to pin down and quantify factorisation-violation effects. 
The structure of the paper is as follows. In Sect. 2, we introduce the case for heavy-ion physics for AFTER@LHC. In Sect. 3, we discuss our simulation set-up and present performance studies for quarkonium and Drell-Yan probes. We conclude in Sect. 4.

\section{Quark-Gluon-Plasma Studies with AFTER@LHC}

\subsection{General Considerations}

One of the main incentives to study relativistic heavy-ion collisions is the unique opportunity to probe highlyexcited and dense nuclear matter under controlled laboratory conditions. If the temperature and density are high enough, Quantum ChromoDymanics (QCD), the theory of the strong interaction, predicts the existence of a new phase of matter, the so-called quark-gluon plasma (QGP), in which most of the quarks and gluons, normally confined within hadrons, are liberated. Lattice-QCD calculations indeed show that there is a rapid rise of the entropy density when the temperature reaches $160 \mathrm{MeV}$. Beyond this temperature the effective number of degrees of freedom saturates near the number of quark and gluon helicity and colour states and the entropy density approaches the Stefan-Boltzmann limit.

Relativistic heavy-ion collisions offer an ideal environment to reach the conditions of the phase transition, both in terms of the necessary temperature and volume for the system thermalisation. Even at moderately high energies such as the ones obtained at the CERN SPS, the average multiplicity in protonproton $(p p)$ collisions at a c.m.s. energy $E_{c . m . s .} \sim 20 \mathrm{GeV}$ is on the order of 3 per unit rapidity with an average momentum close to $0.5 \mathrm{GeV} / c$. This leads to a volume for the system of approximately $1 \mathrm{fm}^{3}$ and an energy density $\varepsilon$ on the order of $0.4 \mathrm{GeV} \mathrm{fm}^{-3}$, that is roughly 3 times the density of the normal nuclear matter. Heavy nucleus-nucleus $(A A)$ collisions, at the same energy per nucleon, rather leads to an energy density of $\varepsilon \sim 2 \mathrm{GeV} \mathrm{fm}^{-3}$ and an initial volume, before the expansion, on the order of 150 $\mathrm{fm}^{3}$.

Among the expected signatures of the QGP formation, quarkonia play an essential role. Since the seminal work of Matsui and Satz [52], the behaviour of quarkonia at high temperature has been considered a signal for deconfinement in heavy-ion collisions. The original idea behind this proposal is that Debye colour screening in a deconfined plasma would reduce the binding of quarks, and thus also affect the formation of heavy-quark bound states. This effect on quarkonia emerged in the QGP would result in the suppression of the quarkonium yields relative to those in the absence of the plasma formation.

The experimental verification of the Matsui-Satz prediction since the late 1980's certainly is one of the most important quests in particle physics in the last 30 years. Huge progress was achieved since then at the SPS, RHIC and LHC in understanding the properties of matter at extreme conditions [53], in particular those produced in high energy proton-nucleus and nucleus-nucleus collisions. Yet, crucial aspects of the resulting system remain unclear. Besides, the mechanism of quarkonium production and suppression in $p p[49,50]$, $p A$ and $A A$ [48] collisions is still far from understood despite significant efforts from the theoretical and experimental sides.

One of the biggest challenge is to find a good baseline to properly use heavy quarkonia to diagnose the QGP. This baseline should allow us to correct the yields for the effects characteristic of heavy-quarkonium production and evolution in hadronic matter when QGP is absent. Such effects, a priori measurable in proton-nucleus collisions, include (1) the modification of the nuclear parton densities, commonly known as shadowing, antishadowing, EMC effect or Fermi motion-depending on the probed kinematics; (2) the multiple scattering of partons or of the heavy-quark pair in the nucleus before or after their creation, which leads to an energy loss or the break-up of the formed quarkonium state; (3) the interaction with other particles produced in the collision-the so-called comovers.

The interplay between these effects and the quarkonium suppression in the QGP is still under debate [48]. Only high precision data on different quarkonium states, in different $p A$ and $A A$ systems and in different kinematics regions $\left(y, P_{T}\right.$ and $\sqrt{s_{N N}}$ ) would clear up these debates.

Another source of complexity indeed results from a possible new charmonium-production mechanism suggested to be at work at high c.m.s. energy. Indeed, $c$ and $\bar{c}$ quarks are so abundantly produced that they could (re)combine into charmonia [48,51,54,55]. In other words, a deconfined nuclear state could lead to more produced $J / \psi$ in some kinematical domains. 


\subsection{The Case for AFTER@LHC}

In this context, it is important to keep in mind the central role played by fixed-target experiments in this field. In general, they provide very high precision measurements whose relevance is clear from the above discussion. The series of experiments NA38, NA50 and NA60 at the CERN SPS program have indeed shown the first tangible signs of anomalous charmonium suppression in heavy-ion collisions [56]. Yet, as of today, this anomalous character could not unambiguously be related to a specific phenomenon or ensemble of phenomena.

Without any surprise, AFTER @LHC, as it happened with these SPS experiments, present significant assets with respect to the present collider experiments, LHC and RHIC. Its c.m.s. energy of $72 \mathrm{GeV}$ allows one to study quarkonium observables in the region of a possible phase transition where charmonium recombination is not expected to play an important role. High luminosities, inherent to the fixed-target mode, are not only necessary to increase the statistical precision but also to provide us with a better control of acceptances and efficiencies. The NA60 experiment together with experiments at HERA and FNAL provide ample proof to that.

Moreover, the NA61 experiment at SPS energies and the RHIC beam-energy-scan program are the natural complement of AFTER@LHC. Together they can provide a much awaited detailed picture of the phase transition region from SPS to RHIC energies and clarify its nature.

Taking into consideration the AFTER @LHC c.m.s. energy and luminosity, the first inclusive high-precision program for quarkonia is at reach. It is also complemented by heavy- and light-flavour studies as we detail now.

\subsubsection{TheAFTER@LHC Quarkonium-Physics Case}

Quarkonium excited states.The scope of AFTER@LHC for quarkonium excited states is fully inclusive and encompasses not only the study of $J / \psi, \psi^{\prime}$ and $\Upsilon(n S)$ states but also the $\chi_{c, b}$ states and the corresponding associated-production channels whose study requires high luminosities, good detector performances (granularity, resolution, PID), wide acceptance and reliable efficiency controls.

It is a known fact that the $J / \psi$ and $\Upsilon(n S)$ states receive significant feed-down contributions. The observed ground states $J / \psi$ and $\Upsilon(1 S)$ are in both cases significantly produced through feed down from higher excited states. This is also true for $\Upsilon(2 S)$ and $\Upsilon(3 S)$. Understanding quarkonium formation and suppression will most probably only be achieved once the excited-state effect are properly accounted for. This begins with careful studies of the $\psi^{\prime}, \Upsilon(2 S)$ and $\Upsilon(3 S)$ yields which is clearly possible with AFTER@LHC.

In Sect. 3, we present our projection for the study of $\psi(n S)$ and $\Upsilon(n S)$ states in $A A$ collisions at AFTER@LHC energies. These complement a previous study [28] for $p p$ and $p A$ collisions.

Yet, a full description of the mechanisms for the formation of the quarkonium $S$ states cannot be dissociated from that of $P$ states. Direct measurements are probably possible with AFTER@LHC with a reduced combinatorial background thanks to the reduced energies compared to the LHC or RHIC. The study of topological properties of the decay distribution such as polarisation [57-59] discussed below can complement such direct studies without requiring to detect photons.

Quarkonium polarisation. Quarkonium polarisation-despite being considered for a long time to be a smoking-gun signal — has so far eluded a thorough explanation despite decades of experimental and theoretical efforts. In the recent years, it was realised that both polar and azimuthal distributions should be measured altogether [58].AFTER@LHC, being a high precision experiment, can play in this respect a very important role in clarifying the evolution of the angular anisotropy parameters for the decay of heavy quarkonia from low to high energies and thus enlighten the production mechanism for these states.

Moreover, as mentioned above, the $\chi_{c}$ and $\chi_{b}$ production in nucleus-nucleus collisions necessarily impacts the interpretation of the $J / \psi$ and $\Upsilon$ suppression as a probe of QGP formation. The observation of the $\chi_{c}$ and $\chi_{b}$ suppression patterns in nucleus-nucleus collisions can help to confirm or falsify the sequential quarkonium melting scenario and, therefore, discriminate between the QGP interpretation and other options.

In addition to a direct observation of the $\chi_{c}$ and $\chi_{b}$ signals in their radiative decays to $J / \psi$ and $\Upsilon(n S)$ which is admittedly challenging in heavy-ion collisions, it may be possible to determine the relative yield of $P$ and $S$ states by only performing dilepton polarisation measurements. The predicted differences in the nuclear dissociation patterns of $S$ and $P$ states-owing to their different binding energies—should result in a change of the observed $J / \psi$ and $\Upsilon$ polarisations from proton-proton to central nucleus-nucleus collisions. This may provide an indication for quarkonium sequential suppression in the QGP. For such observations to be conclusive, the impact of other QGP- and nucleus-related phenomena on the $J / \psi$ and $\Upsilon$ polarisation should 
carefully be investigated alongside in $p A$ collisions or on the states receiving few or no feed down, like the $\psi^{\prime}$ for instance.

\subsubsection{Open Charm and Beauty Production}

The study of $D$ mesons is a natural continuation of the study of $J / \psi$ and $\psi^{\prime}$ formation and dissociation. $D$ mesons have been proposed [60] as a baseline to understand QGP effects on quarkonium. In particular, it was proposed to measure their yield in $p A$ and $A A$ collisions versus rapidity to get some insights on CNM affecting heavy-quark production, which can be common to quarkonium production. Such studies can be complemented with the measurements of azimuthal anisotropies as a function of rapidity to constraint transport properties of the QGP such as the shear viscosity and the heavy-quark diffusion coefficients. The measurement of the nuclear-modification factor, $R_{A A}$ for open-heavy flavour hadrons can also provide insights into the nature of the heavy-quark interaction with the surrounding matter. The data collected at RHIC at the energy of $\sqrt{s_{N N}}=200 \mathrm{GeV}[61,62]$ show a strong suppression at transverse momenta larger than $3 \mathrm{GeV} / c$. These results can be considered as the evidence of heavy-quark energy loss in the hot and dense QGP. However, the details of the interactions with the QGP are not well understood; the major difficulty is to determine the role of the gluon radiation (radiative energy loss) and of the collisional energy loss (due to collisions with other objects in the QGP). A few different models with distinctive assumptions describe reasonably well the current data [61]. New observables_-preferably for open charm and beauty separately - are thus required to constrain these theoretical approaches.

AFTER@LHC will be capable of delivering high-quality data to address this issue. The measurements of nuclear modification factors for D mesons as a function of the transverse momentum and rapidity with AFTER@LHC will provide extremely valuable inputs since the radiative and collisional energy loss are expected to exhibit different $p_{T}$ and $y$ dependencies [22]. These high statistics data will also facilitate studies of heavy-flavour azimuthal correlations $(D-D, J / \psi-D)$ which will give another handle on heavy-quark in-medium interactions. These will indeed allow for precise determinations of the transport properties of the QGP, including the energy-loss transport coefficient $\hat{q}$ and the charm-quark-diffusion coefficients. Finally, the measurements of the nuclear-modification factor for $b$-hadrons in $p A$ interactions will be measurable with a good precision [22,28]. $b$-hadron $R_{A A}$ measurements via non-prompt $J / \psi$ in $\mathrm{Pb} A$ collisions will also be at reach with a slightly reduced statistics compared to the prompt ones but with a smaller background.

\subsubsection{Drell-Yan}

The hadro-production of a large invariant-mass lepton pair, known as the Drell-Yan (DY) process, is a clean, precise and controllable probe of the short-distance dynamics and the partonic structure of hadrons. In particular, the DY process on nuclear targets is an ideal tool to quantify the initial-state effects taking place during such proton-nucleus reactions. Let us cite the nuclear modification of the parton densities or coherence effects. Indeed, the production of the lepton pair by an electroweak gauge boson does not suffer from final-state interactions, typically associated with the phenomena of energy loss or absorption.

Beside being a typical background for the study of quarkonia, it is also an important process per se where the factorisation of initial-state nuclear effects can be tested. With a nuclear target, the AFTER@LHC kinematic with a LHCb-like detector cover $2 \lesssim y_{\text {lab }} \lesssim 5$ correspond to the antishadowing region for the parton in the $\operatorname{targer}\left(x_{2} \in[0.05: 0.2]\right)$ for invariant masses slightly above the charmonium family. Increasing the dilepton mass allows one to probe the EMC region, where the nuclear modification of the PDFs was historically observed for the first time. The use of $\mathrm{Pb}$ beam allows one to probe a region where shadowing effects are expected. Overall, the study of $p A, \mathrm{~Pb} p$ and $\mathrm{Pb} A$ collisions altogether will offer an unique playground to test the factorisation of nPDF effects.

\subsubsection{Soft-Probe Studies}

The aforementioned AFTER@LHC studies on hard probes will be complemented with that of soft probes in heavy-ion collisions. In fact, the bulk of low-momentum particles produced in $A A$ collisions at RHIC and at the LHC is supposed to originate from a fluid-like medium, whose transverse expansion is largely driven by the density gradients produced in the earliest stage of the collision $[63,64]$. The harmonic analysis of particle production in $A A$ collisions typically focuses on the Fourier decomposition of the azimuthal angle $\phi$ dependence over a narrow region in pseudorapidity around $\eta=0$. The $v_{n}$ coefficients of this decomposition 


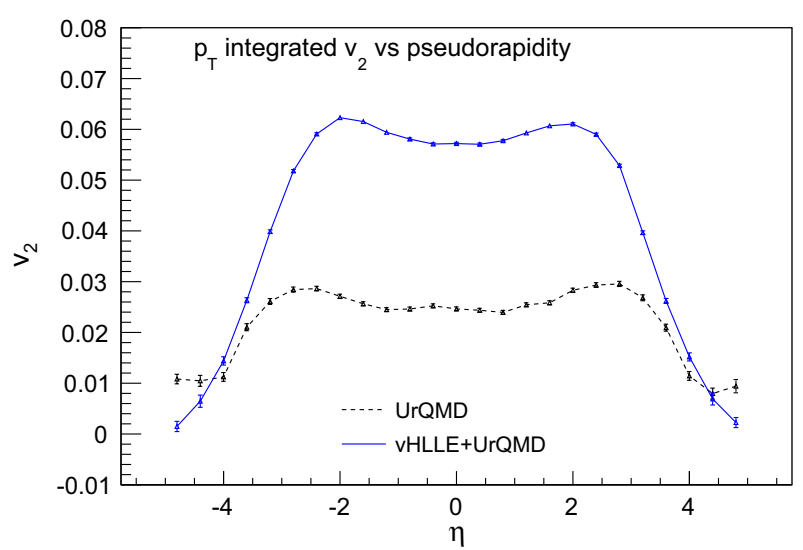

Fig. $1 p_{T}$-integrated $\left(p_{T}\right.$ range $\left.=[0.2,2.0] \mathrm{GeV}\right)$ elliptic flow (calculated using event plane method) of all charged hadrons as a function of c.m.s. pseudorapidity in PbPb collisions at $\sqrt{s_{N N}}=72 \mathrm{GeV}$ and centrality 20-30\% (which in the model correspond to impact parameter range $b=[6.8,8.3] \mathrm{fm}$ ). The dashed curve corresponds to a pure cascade case $(U r Q M D)$ and is compared to the case (solid blue curve) with an intermediate hydrodynamic phase of the evolution $v H L L E+U r Q M D$ (hydro + cascade model) [66]

play an important role in understanding the collective behavior of the system. The study of both even and odd order coefficients [65] provide tight constraints on the collective dynamics and should be determined for that reason.

This requires, in particular, a baseline comparison of $v_{2}$ (and $v_{n}$ ) to existing models in $A B$ collisions, for two otherwise opposite assumptions - with and without a hydrodynamic phase of the evolution. Figure 1 shows predictions of charged hadrons $v_{2}$ versus pseudorapidity in semi-central $\mathrm{PbPb}$ collisions at $\sqrt{s_{N N}}=$ $72 \mathrm{GeV}$ for a pure cascade (UrQMD) case and for the case when a hydrodynamic evolution is included (vHLLE+UrQMD) [66]. AFTER @LHC will be able to continue the existing studies on $v_{n}$ at energies between the RHIC Beam Energy Scan $(B E S)$ and the top RHIC and LHC energies not only with high precision but also in a broad rapidity range. Doing so, it will provide a detailed account of the collective evolution between the two energy ranges, around the expected transition line in the QCD phase diagram, between the existing high precision SPS results and the LHC.

\subsubsection{Going Further}

- During the ramp up in energy of the LHC, AFTER@LHC can perform an energy scan to search for a possible critical point along the phase transition line, complementing the RHIC BES program. Even if this scan may be an experimental challenge owing to the possible beam instabilities during this phase, it can provide relatively high statistics allowing for direct searches of the critical point and to look for phasetransition signatures. As for the soft probes study at $72 \mathrm{GeV}$, it would be complementary to the RHIC BES II program.

- A study of the chiral symmetry restoration à la NA60 can be performed in AFTER @LHC by studying the dimuon mass spectrum in $A A$ for the $\rho, \omega, \phi$ systems. Different measurements can be performed, namely the change in the branching ratio between leptonic and hadronic channels and the change in the width of the mass peaks. This requires significant statistics, a high resolution and a careful control of the background processes including the charm contribution. A comparison with the STAR observation at RHIC (excess with $2 \sigma$ at $\sqrt{s_{N N}}=62 \mathrm{GeV}$-without charm background subtraction) would be extremely useful. Since the NA60 results [67] were obtained with a resolution of $20 \mathrm{MeV}$ at the $\omega$ and $\phi$ peaks and AFTER @LHC studies should aim at such precision to be competitive. The particle identification which could be done with a LHCb-like detector would also considerably simplify the analysis and overcome many of the drawbacks present in NA60.

Overall, AFTER@LHC can considerably enrich the existing studies devoted to the search for signatures of the chiral symmetry restoration which plays a fundamental role in explaining the origin of $95 \%$ of the meson and baryon masses. Aided by lattice studies and a considerable wealth of information collected since decades on the fundamental aspects of chiral symmetry in the strong sector [68], AFTER @ LHC is in a unique position 
to clarify the role of the chiral-symmetry restoration in the dynamics of meson and baryon interactions and also its importance in the interplay between chiral-symmetry restoration and deconfinement transition [69].

Finally, AFTER@LHC, due to its extended kinematic range can scan a much larger chemical baryon potential range in the QGP phase diagram compared to measurements at mid-rapidity which are presently available in most of the experimental results.

\section{Simulations}

In this section, we present feasibility studies for quarkonium and Drell-Yan production via the di-muon decay channels in heavy-ion collisions with a $2.76 \mathrm{TeV}$ per nucleon LHC lead beam on a fixed target $\left(\sqrt{s_{N N}}=72 \mathrm{GeV}\right)$. In Ref. [28], we have presented simulations for $p p$ collisions at $\sqrt{s}=115 \mathrm{GeV}$ resulting from $7 \mathrm{TeV}$ protons impinging on a hydrogen target with a $\mathrm{LHCb}$-like detector. In the following, we follow a similar procedure which we have updated for the heavy-ion case as described below. In addition, we elaborate a little on the possibilities offered by the ALICE detectors used in the fixed-target mode.

\subsection{Simulation Framework}

The simulations presented in this paper are performed for $p p$ collisions at $\sqrt{s_{N N}}=72 \mathrm{GeV}$. Projections for $p A$ and $A A$ collisions at $\sqrt{s_{N N}}=72 \mathrm{GeV}$ are done by applying a nuclear scaling factor to the $p p$ simulations, assuming neither nuclear nor isospin effects.

Different di-muon sources are separately studied in order to keep a good control over the input distributions and the normalization of these different sources. As a signal we consider either quarkonia or Drell-Yan (which is a background in the case of quarkonium studies). The background under the signal is twofold: the correlated background-charm and beauty pair production $\left(c+\bar{c} \rightarrow D^{+}+D^{-} \rightarrow l^{+} l^{-}\right.$and $\left.b+\bar{b} \rightarrow B^{+}+B^{-} \rightarrow l^{+} l^{-}\right)$, and the combinatorial background, mainly muons from pion and kaon decays.

The quarkonium signals as well as the Drell-Yan and charm- and beauty-pair production $(c \bar{c}$ and $b \bar{b})$ are simulated with HELAC- ONIA [70,71] which provides Les Houches Event Files [72] as output. These events are then processed with PYтнIA 8 [73] to perform the hadronisation, to account for initial/final-state radiations and to decay the resonances. At this step, additional muons from the underlying PYтнIA event can be produced. We have checked that the contribution of combinations of these muons with muons from initial quarkonia or $c \bar{c}$, $b \bar{b}$ pairs is negligibly small and thus is not included in the correlated background.

$J / \psi, \psi^{\prime}, \Upsilon$ states and the $c \bar{c}$ continuum rates are obtained in a data-driven way as in [28]. Quarkonia processed in PYтнIA 8 are forced to decay into the dimuon-decay channel and the simulated yields are then weighted by the corresponding cross section multiplied by the Branching Ratio (BR) [74]. The open-beauty simulation is performed with a Leading Order (LO) matrix element normalised to a Next-To-Leading-Order (NLO) $K$ factor found to be 1.83 [28]. The Drell-Yan simulation is performed with the process $q \bar{q} \rightarrow \gamma^{\star} / Z \rightarrow$ $\mu^{+} \mu^{-}$at LO, with the CTEQ6L1 pdf set. In this case, the $K$ factor is found to be 1.2. In order to decrease the time for the simulation in the di-muon invariant mass $\left(M_{\mu^{+} \mu^{-}}\right)$region of interest, the simulation is done with a $M_{\mu^{+} \mu^{-}}>1.5 \mathrm{GeV} / c^{2}$ requirement.

The combinatorial background is obtained from minimum bias $p p$ collisions generated with PYTHIA 8 , using the process SoftQCD: nonDiffractive with the MRSTMCal.LHgrid LHAPDF (6.1.4) set [75]. The dominant source of combinatorial opposite-sign di-muon pairs are $\mu^{+/-}$coming from $\pi^{+/-}$or $K^{+/-}$ decays.

The PүтніA 8 output is then processed via a fast simulation framework. This last step is performed to account for realistic detector-resolution and particle-identification performances. The detector responsemomentum resolution, $\mu$ identification efficiency and $\pi / K$ misidentification probability with $\mu$-is simulated with a detector setup similar to the LHCb detector [76] with a pseudorapidity coverage of $2<\eta_{\text {lab }}<5$. The minimum transverse momentum of single muons is required to be greater than $0.7 \mathrm{GeV} / c$. The considered momentum resolution is taken as $\delta p / p=0.5 \%$, LHCb reporting: $\delta p / p \sim 0.4(0.6) \%$ for a momentum of 3 (100) GeV/c [77]. The considered single $\mu$ identification efficiency of $\epsilon_{\mu^{+/}}=98 \%$ is an average LHCb efficiency for muons coming from $J / \psi$ decays, for $p>3 \mathrm{GeV} / c$ and $p_{\mathrm{T}}>0.8 \mathrm{GeV} / c$ [77]. In the case of muons from hadronic decays, the muon is not considered in the simulation if a $\pi / K$ decays before $12 \mathrm{~m}$. This corresponds to a decay before the LHCb calorimeter which can be rejected by the LHCb tracking system. If the $\mu$ is produced beyond $12 \mathrm{~m}$ or if a $\pi / K$ is misidentified with $\mu$ in the muon stations, the following 
Table 1 Total cross section for different processes in $p p$ collisions at $\sqrt{s}=72 \mathrm{GeV}$ obtained from HELAC- ONIA simulations, and in the case of the minimum bias simulation from PYTHIA 8

\begin{tabular}{lc}
\hline & $\sigma_{\text {tot }}(\mathrm{mb})$ \\
\hline$J / \psi$ & $5.51 \times 10^{-4}$ \\
$\psi^{\prime}$ & $5.90 \times 10^{-5}$ \\
$\Upsilon(1 \mathrm{~S})$ & $6.87 \times 10^{-7}$ \\
$\Upsilon(2 \mathrm{~S})$ & $1.82 \times 10^{-7}$ \\
$\Upsilon(3 \mathrm{~S})$ & $7.56 \times 10^{-8}$ \\
Drell-Yan $\left(\mathrm{M}>1.5 \mathrm{GeV} / c^{2}\right)$ & $6.66 \times 10^{-6}$ \\
$c \bar{c}$ & $1.07 \times 10^{-1}$ \\
$b \bar{b}$ & $9.44 \times 10^{-5}(g g \rightarrow b \bar{b})$ \\
Minimum bias & $5.86 \times 10^{-5}(q \bar{q} \rightarrow b \bar{b})$ \\
\hline
\end{tabular}

momentum-dependent $\pi / K$ misidentification probabilities [78] are applied: $P_{M I D}(\pi \rightarrow \mu)(p)=(0.5+$ $6.63 \exp (-0.13 p)) \%$ and $P_{M I D}(K \rightarrow \mu)(p)=(0.5+8.6 \exp (-0.11 p)) \%$, for $\pi$ and $\mathrm{K}$ respectively. The di-muon efficiency is taken as: $\epsilon_{\mu^{+} \mu^{-}}=\epsilon_{\mu^{+}} \times \epsilon_{\mu^{-}}$. It is assumed in these simulations that the detector performance does not decrease with the event multiplicity.

Projections for $p A$ and $A A$ collisions are done by applying a nuclear scaling factor, accounting for the number of binary collisions, to the cross sections obtained from the $p p$ simulations. Then, the signal and background sources are normalised to the desired integrated luminosity according to the production cross section of the process (taking into account the initial phase space cuts, if any). The values of the cross sections that are obtained from the $p p$ simulations at $\sqrt{s_{N N}}=72 \mathrm{GeV}$ are reported in Table 1 , they are integrated over rapidity and $p_{T}$. In the case of quarkonium, Drell-Yan, open charm and beauty simulations, the nuclear scaling factor for minimum-bias $p A$ collisions is $A$ and for $A B$ collisions it is $A B$, where $A$ and $B$ are the beam and projectile mass numbers, respectively. These scaling factors correspond to the absence of hot/cold nuclear and isospin effects. The scaling factor for the combinatorial background is $A \times N_{\text {coll }}^{p A}$ and $A B \times N_{\text {coll }}^{A B}$ for $p A$ and $A B$ collisions, respectively, where $N_{\text {coll }}$ is an average number of binary collisions obtained from Glauber simulations $[22,79,80]$ for minimum-bias collisions for a given colliding system.

In what follows, we will study the case of Xe gas target, one of the heavier noble gases. The LHC heavy-ion runs are expected to last for about a month per year, which we take as $10^{6} \mathrm{~s}$. In order to provide a baseline at the same energy, $2.76 \mathrm{TeV}$ proton beam can be delivered for about a week per year, and our estimated running time for proton-beam collisions on a hydrogen gas target is $0.25 \times 10^{6} \mathrm{~s}$. The expected instantaneous and yearly (as defined before) luminosities for $p p$ and $\mathrm{PbXe}$ collisions at $\sqrt{s}=72 \mathrm{GeV}$ are gathered in Table 2. A month of running with the $\mathrm{LHC} \mathrm{Pb}$ beam on a gas target provides an access to heavy-ion studies with a yearly integrated luminosity as high as $30 \mathrm{nb}^{-1}$ with an integrated luminosity of the $p p$ baseline reaching $1.5 \mathrm{fb}^{-1}$. Furthermore, studies of $p A$ collisions at the same centre-of-mass energy of $\sqrt{s}=72 \mathrm{GeV}$ would be of particular interest. The instantaneous luminosity for $p A$ collisions (with a $\mathrm{Xe}, \mathrm{Kr}, \mathrm{Ar}$ or $\mathrm{Ne}$ gas target) is included in Table 2. These instantaneous luminosities are expected to be achieved with a 1-m-long HERMES-like internal gas target [21]. Such an open-storage-cell system with a dedicated pumping system offers a possibility to reach a higher gas pressure. ${ }^{1}$ Given these high instantaneous luminosities, one can obtain the same yields of $J / \psi$, $\psi^{\prime}, \Upsilon$ and Drell-Yan in $p A$ collisions as in the $p p$ case with relatively short runs. For example, in the case of the $p \mathrm{Xe}$ system considered in this paper, a running time of $13 \mathrm{~h}$ is enough, which corresponds to an integrated luminosity of $\sim 2 \mathrm{pb}^{-1}$. For $\mathrm{Kr}$, Ar and $\mathrm{Ne}$ cases that would be 20,42 and $84 \mathrm{~h}$ of running, respectively. These running times correspond to integrated luminosities of $\sim 3,6.5$ and $13 \mathrm{pb}^{-1}$ for $p \mathrm{Kr}, p \mathrm{Ar}$ and $p \mathrm{Ne}$ collisions, respectively. ${ }^{2}$ Table 2 also includes the average number of binary collisions for minimum-bias events, for each of the considered colliding systems: $p p, p$ Xe and PbXe.

The projections which are presented here for $p$ Xe collisions give the highest combinatorial background out of the mentioned $p \mathrm{Xe}, p \mathrm{Kr}, p \mathrm{Ar}, p \mathrm{Ne}$ cases with the $2.76 \mathrm{TeV}$ proton beam, due to the highest number of binary collisions. As a $p p$ reference, the $\mathrm{H}_{2}$ target is considered since it allows to reach a higher instantaneous luminosity and the target polarisation is not required for the heavy-ion studies. The important advantage of the

\footnotetext{
${ }^{1}$ In [28], a SMOG-like system was considered and resulted in lower instantaneous luminosities.

2 The mentioned instantaneous luminosities correspond to an inelastic rate of $400 \mathrm{MHz}$. This may however be limited by detector capabilities. In such case, longer running times may be needed in order to achieve the quoted integrated luminosities for $p A$ systems
} 
Table 2 Expected instantaneous and yearly (as described in the text) luminosities for $\mathrm{Pb} A, p A$ and $p p$ collisions with $2.76 \mathrm{TeV}$ beams, and average number of binary collisions for $\mathrm{PbXe}$ and $p \mathrm{Xe}$ minimum bias collisions from the Glauber Monte-Carlo calculations

\begin{tabular}{lllll}
\hline Beam & Target gas & $\mathcal{L}\left[\mathrm{cm}^{-2} \mathrm{~s}^{-1}\right]$ & $\int d t \mathcal{L}$ & $\left\langle N_{\text {coll }}\right\rangle$ \\
\hline $\mathrm{Pb}$ & $\mathrm{Xe}(\mathrm{Kr}, \mathrm{Ar}, \mathrm{Ne})$ & $3 \times 10^{28}$ & $30 \mathrm{nb}^{-1}$ & 165.1 \\
$p$ & $\mathrm{Xe} \mathrm{Kr}, \mathrm{Ar}, \mathrm{Ne}$ & $2.34 \times 10^{32}$ & $2 \mathrm{pb}^{-1}, 3 \mathrm{pb}^{-1}, 6.5 \mathrm{pb}^{-1}, 13 \mathrm{pb}^{-1}$ & 3.7 \\
$p$ & $\mathrm{H}$ & $0.92 \times 10^{33}$ & $250 \mathrm{pb}^{-1}$ & 1 \\
$p$ & $\mathrm{H}_{2}$ & $5.8 \times 10^{33}$ & $1.5 \mathrm{fb}^{-1}$ & 1 \\
\hline
\end{tabular}

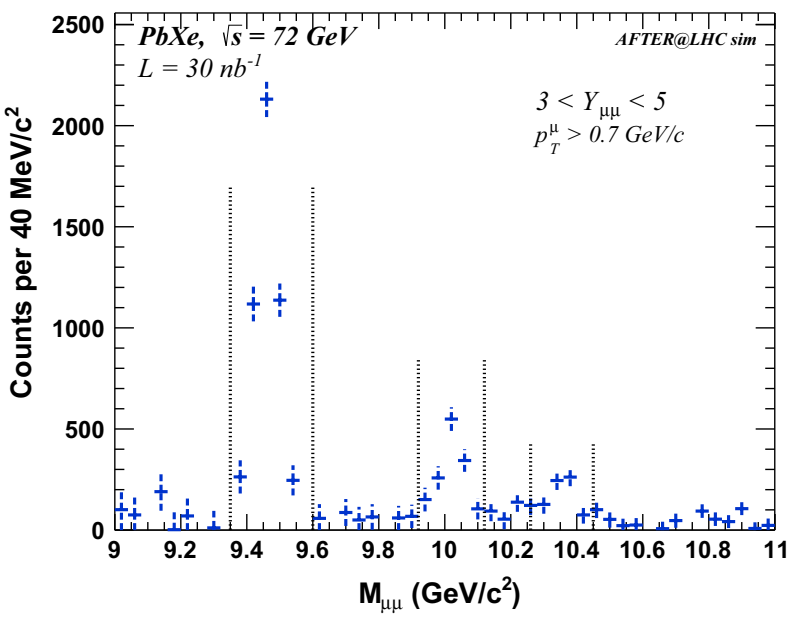

Fig. $2 \Upsilon(n S)$ signal after the (like-sign) combinatorial-background subtraction with the expected statistical uncertainties in PbXe collisions at $\sqrt{s_{N N}}=72 \mathrm{GeV}$ in $3<y_{\mathrm{lab}}<5$ for $\int \mathcal{L}_{\mathrm{PbXe}}=30 \mathrm{nb}^{-1}$, assuming $R_{A A}=1$

fixed-target mode is the possibility to change relatively easily from one target to another. This would allow us to study different systems, such as $\mathrm{Pb} A, \mathrm{~Pb} p$ and $p A$ collisions at the same energy, in different years of LHC running.

\subsection{Quarkonium Studies at $\sqrt{s_{N N}}=72 \mathrm{GeV}$}

For the quarkonium $\mathrm{PbXe}$ simulations where the full background is considered, the combinatorial one is subtracted assuming the like-sign technique. The background can be reconstructed as an arithmetic or geometric mean of all $\mu^{+} \mu^{+}$and $\mu^{-} \mu^{-}$pairs produced in an event. That is the worst-case scenario in terms of the expected statistical uncertainties from the combinatorial-background subtraction: the statistical uncertainties of the background scale as the background yield under the signal $\left(N_{b k g}\right)-\sqrt{N_{b k g}}$-and add to that of the signal when subtracted. However, this is the most straightforward method of extracting the combinatorial background in an experiment. It does not require an additional normalisation factor and thus does not suffer from additional systematic uncertainties, as the event-mixing technique for example.

As can be seen on Fig. 2, high $\Upsilon(n S)$ yields are expected in PbXe heavy-ion collisions with an integrated luminosity of $30 \mathrm{nb}^{-1}$. The plot shows the di-muon invariant-mass distribution in the $\Upsilon(n S)$ mass range after the combinatorial background subtraction with a like-sign technique assuming $R_{A A}=1$. Thanks to the large $\Upsilon(n S)$ yields and large enough signal/background ratios $(S / B)$, each of the $\Upsilon$ state- $\Upsilon(1 S), \Upsilon(2 S)$ and $\Upsilon(3 S)$ - can easily be reconstructed. The dashed lines represent expected width of $\Upsilon(n S)$ states assuming the LHCb-like single muon $p_{T}$ resolution: the $\Upsilon$ states can clearly be separated. The other background sources, namely correlated $b \bar{b}$ and Drell-Yan pairs, are negligibly small in the $\Upsilon(n S)$ invariant-mass range. The expected yields for $\Upsilon(1 S), \Upsilon(2 S)$ and $\Upsilon(3 S)$ and $S / B$ ratios are summarised in Table 3 for $p p$, $p$ Xe and $\mathrm{PbXe}$ collisions. They are integrated over the $\Upsilon p_{T}$ and a rapidity range of $3<y_{\text {lab }}<5$, accessible for the $\Upsilon$ production. The $p_{T}$ and rapidity distributions, for each $\Upsilon$ state are shown in Fig. 3.

With the integrated luminosity of $30 \mathrm{nb}^{-1}$, extremely large $J / \psi$ and $\psi^{\prime}$ yields are expected. The $J / \psi$ and $\psi^{\prime} p_{T}, d N / d p_{T}$, and rapidity, $d N / d y_{\text {lab }}$, distributions are shown in Fig. 3, assuming $R_{A A}=1 . J / \psi$ and $\psi^{\prime}$ 
Table $3 \Upsilon(n S)$ yields and $\Upsilon(n S)$ signal over the combinatorial background ratios $(S / B)$ for $p p$, $p$ Xe and PbXe collisions at $\sqrt{s_{N N}}=72 \mathrm{GeV}$ and $3<y<5, \int \mathcal{L}_{p p}=1.5 \mathrm{fb}^{-1}, \int \mathcal{L}_{p \mathrm{Xe}}=2 \mathrm{pb}^{-1}, \int \mathcal{L}_{\mathrm{PbXe}}=30 \mathrm{nb}^{-1}$

\begin{tabular}{llll}
\hline Yields & & Signal & S/B \\
\hline$\Upsilon(1 S)$ & $p p$ & $7.95 \times 10^{3}$ & 29.0 \\
& $p \mathrm{Xe}$ & $1.39 \times 10^{3}$ & 7.8 \\
& $\mathrm{PbXe}$ & $4.33 \times 10^{3}$ & $1.8 \times 10^{-1}$ \\
$\Upsilon(2 S)$ & $p p$ & $1.75 \times 10^{3}$ & 8.2 \\
& $p \mathrm{Xe}$ & $3.06 \times 10^{2}$ & 2.2 \\
& $\mathrm{PbXe}$ & $9.56 \times 10^{2}$ & $5.0 \times 10^{-2}$ \\
$\Upsilon(3 S)$ & $p p$ & $8.25 \times 10^{2}$ & 10.3 \\
& $p \mathrm{Xe}$ & $1.44 \times 10^{2}$ & 2.8 \\
& $\mathrm{PbXe}$ & $4.49 \times 10^{2}$ & $6.2 \times 10^{-2}$ \\
\hline
\end{tabular}
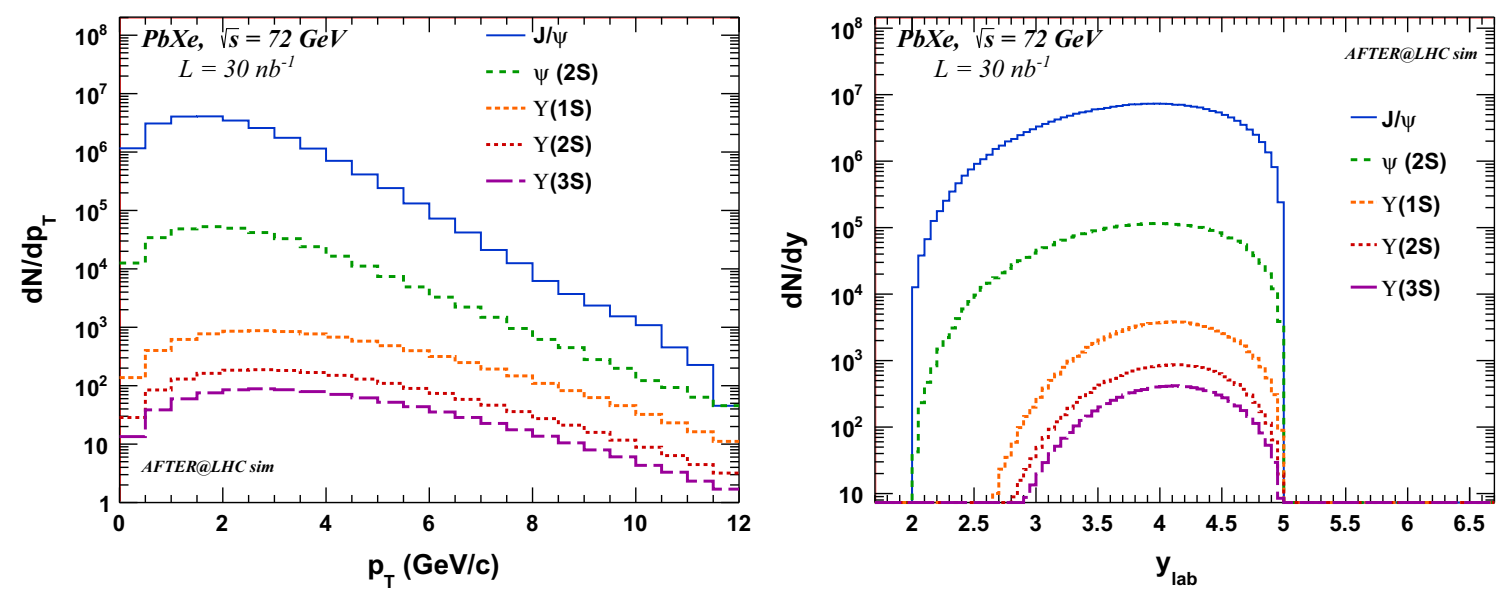

Fig. $3 J / \psi, \psi^{\prime}, \Upsilon(1 S), \Upsilon(2 S)$ and $\Upsilon(3 S) p_{T}, d N / d p_{T}$, (left) and rapidity, $d N / d y_{\text {lab }}$, (right) distributions in PbXe collisions at $\sqrt{s_{N N}}=72 \mathrm{GeV}$ for $\int \mathcal{L}_{\mathrm{PbXe}}=30 \mathrm{nb}^{-1}$, assuming $R_{A A}=1$

signals can be studied with a good precision over a wide transverse momentum range, up to $\sim 12 \mathrm{GeV} / c$, and in the rapidity range limited only by the detector acceptance.

The left panel of Fig. 4 shows the di-muon invariant-mass distribution in the $J / \psi$ and $\psi^{\prime}$ mass region and in the entire rapidity range of $2<y_{\text {lab }}<5$. As in the $\Upsilon$ case, the combinatorial background is subtracted using the like-sign technique. In addition, the contributions from correlated $c \bar{c}, b \bar{b}$ and Drell-Yan backgrounds are included. The $c \bar{c}$ correlated background dominates over the other background sources, under the $J / \psi$ and $\psi^{\prime}$ signal peaks.

The right panel of Fig. 4 shows our predicted statistical precision for the nuclear-modification factors $\left(R_{\mathrm{PbXe}, p \mathrm{Xe}}\right)$ for $J / \psi$ (red markers) and $\psi^{\prime}$ (blue markers) in PbXe (filled symbols) and $p \mathrm{Xe}$ (open symbols) collisions at $\sqrt{s_{N N}}=72 \mathrm{GeV}$. The statistical uncertainties include that of the $p p$ baseline uncertainties of the $J / \psi$ and $\psi^{\prime}$ expected yields and of the like-sign combinatorial background subtraction-which is the dominant source here. $R_{\mathrm{PbXe}, p \mathrm{Xe}}$ can be extracted as a function of rapidity, in three $y$ ranges, with a very good precision.

Such measurements of quarkonia in wide rapidity ranges will allow one to probe the hot and dense medium resulting from these heavy-ion collisions at different temperatures. Furthermore, to gain even more information on the medium properties, such high $J / \psi$ and $\psi^{\prime}$ expected yields would be used for analyses of $R_{A A}$ differential in centrality, as well as measurements of the elliptic flow, $v_{2}$, which we leave for a future publication.

\subsection{Drell-Yan Pair Production in Heavy-Ion Collisions at $\sqrt{s_{N N}}=72 \mathrm{GeV}$}

The physics program of AFTER also includes the precise measurement of the Drell-Yan pair-production process which can probe initial-state effects on quarks using different $\mathrm{Pb} A$ collision species. 

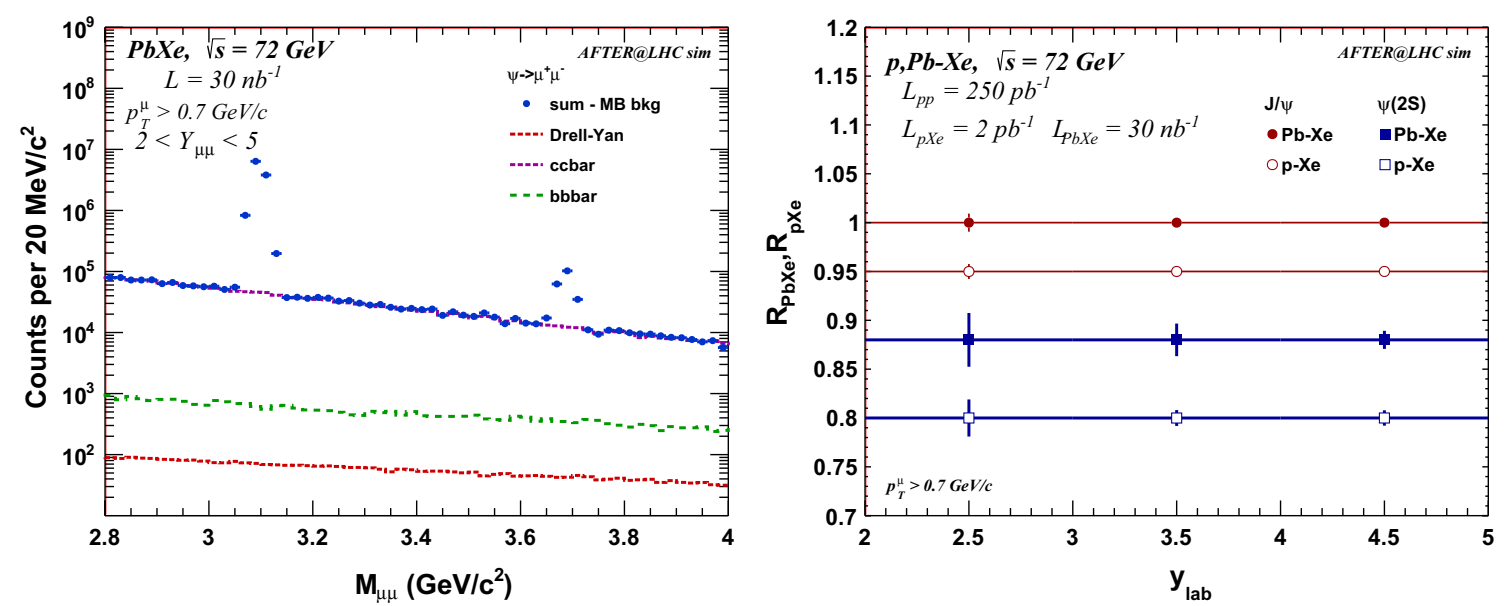

Fig. 4 Left $J / \psi$ and $\psi^{\prime}$ signals after the (like-sign) combinatorial-background subtraction with the expected statistical uncertainties, $\mathrm{PbXe}$ collisions at $\sqrt{s_{N N}}=72 \mathrm{GeV}$ in $2<y_{\mathrm{lab}}<5$ and $\int \mathcal{L}_{\mathrm{PbXe}}=30 \mathrm{nb}^{-1}$, assuming $R_{A A}=1$. Right Statistical precision of the nuclear modification factors $\left(R_{\mathrm{PbXe}}\right.$ and $\left.R_{p \mathrm{Xe}}\right)$ versus rapidity in $\mathrm{PbXe}$ and $p \mathrm{Xe}$ at $\sqrt{s_{N N}}=72 \mathrm{GeV}$, assuming the combinatorial background subtraction with the like-sign technique. The integrated luminosities are: $\int \mathcal{L}_{p p}=1.5 \mathrm{fb}^{-1}, \int \mathcal{L}_{p \mathrm{Xe}}$ $=2 \mathrm{pb}^{-1}, \int \mathcal{L}_{\mathrm{PbXe}}=30 \mathrm{nb}^{-1}$, for $p p, p \mathrm{Xe}$ and $\mathrm{PbXe}$ respectively

A great challenge in the Drell-Yan measurement at high energies is the large correlated background from $c+\bar{c} \rightarrow D^{+}+D^{-} \rightarrow l^{+} l^{-}$and $b+\bar{b} \rightarrow B^{+}+B^{-} \rightarrow l^{+} l^{-}$. This background is smaller at the c.m.s. energy of AFTER @LHC in $\mathrm{Pb} A$ collisions, and can easily be further reduced with a modern vertex detector by applying a secondary vertex cuts on displaced production of $D$ and $B$-mesons. Figure 5 separately shows the expected yields for Drell-Yan, $c \bar{c}$ and $b \bar{b}$ production in PbXe collisions as a function of the di-muon invariant mass in the range between the $\psi$ and $\Upsilon\left(4<M_{\mu^{+} \mu^{-}}<8 \mathrm{GeV} / c^{2}\right)$, for different rapidity ranges within $2<y_{\text {lab }}<5$. A single muon $p_{T}^{\mu}$ cut of $1.2 \mathrm{GeV} / c$ is applied. We also stress here that going to the very backward rapidity region $\left(2<y_{\text {lab }}<3\right)$ - which is probably the most interesting one physics wise-renders the Drell-Yan signal even cleaner since the quark-induced processes are favoured and the background is reduced accordingly.

Due to the continuous nature of the signal di-muon invariant-mass distribution shape and to the reduced $S / B$ ratios, the like-sign technique does not provide enough statistical precision for the signal extraction in this case. However, one can precisely determine the high combinatorial background expected in $\mathrm{Pb} A$ collisions with the event-mixing technique. ${ }^{3}$ This technique combined with a large amount of like-sign di-muon background pairs (used to normalise the shape determined by the mixed events) should provide a robust subtraction of the combinatorial background even for small $S / B$ values. These expected Drell-Yan yields and $S / B$ ratios are gathered in Table 4. In such case, the main remaining uncertainty would be of systematical origin, thus difficult to simulate. As such, the combinatorial background is not accounted for in the distributions shown in Fig. 5, which should therefore be considered as idealised. On the way, we note that the event-mixing technique can also be applied to improve the extraction of the quarkonium-signal mentioned above.

We note that the $S / B$ also improves with increasing $M_{\mu^{+}} \mu^{-}$and when going to the most backward rapidity region. This is clear from Table 4 and Fig. 6, where the signal over the combinatorial-background ratio is studied as a function of the di-muon invariant mass for the integrated rapidity region of $2<y_{\text {lab }}<5$, and for the following 3 ranges: $2<y_{\text {lab }}<3,3<y_{\text {lab }}<4$ and $4<y_{\text {lab }}<5$. As can be also seen on Fig. 6 , the S/B can be increased by increasing the $p_{T}$ cut on a single muon. In order to maximise the $S / B$, the single muon minimum $p_{T}^{\mu}$ cut is changed from 0.7 to $1.2 \mathrm{GeV} / c$. Indeed the particles that contribute to the combinatorial background are mostly produced with lower $p_{T}$. However, a further increase of the minimum muon $p_{T}^{\mu}$ starts to impact the Drell-Yan signal.

\subsection{Acceptances of the ALICE Detectors in the Fixed-Target Mode}

The ALICE experiment is designed to cope with the high multiplicity of heavy-ion collisions and a fixed-target mode can also be implemented for this experiment. The ALICE detector consists in a central barrel part, which

\footnotetext{
3 where an arbitrarily large number of $\mu^{+}$and $\mu^{-}$from different events can be mixed and paired.
} 

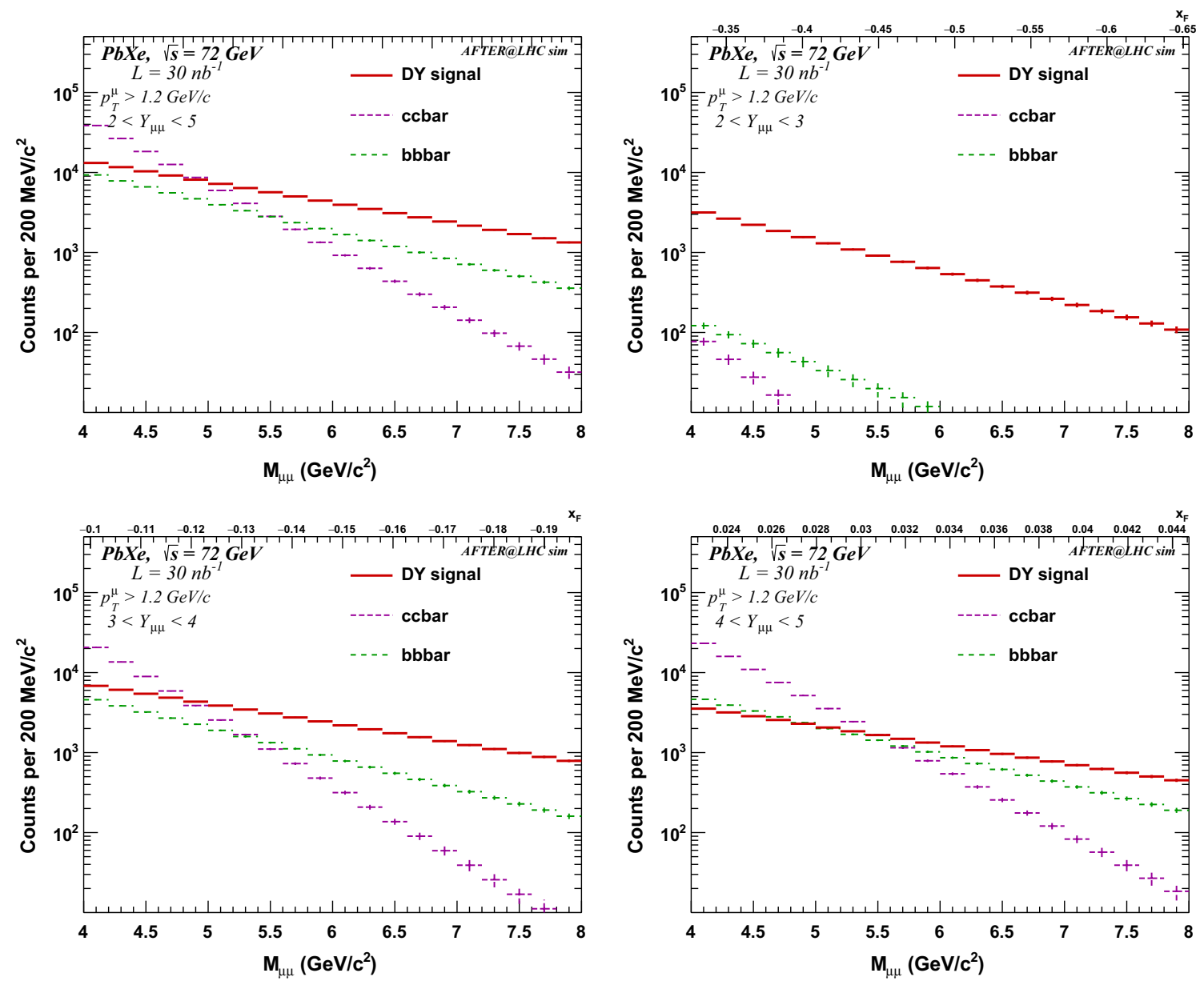

Fig. 5 Di-muon invariant-mass distributions $\left(4<M_{\mu^{+} \mu^{-}}<8 \mathrm{GeV} / c^{2}\right)$ for Drell-Yan, $c \bar{c}$ and $b \bar{b}$ productions, in the integrated rapidity range of $2<y_{\text {lab }}<5$ (top left) and divided into following ranges: $2<y_{\text {lab }}<3$ (top right), $3<y_{\text {lab }}<4$ (bottom left) and $4<y_{\text {lab }}<5$ (bottom right). The upper $x$ axis represents the corresponding $x_{F}$ values in a given rapidity range and invariant-mass bin. The combinatorial background is not presented and systematic uncertainties resulting from the background subtraction with with the event-mixing technique are not included. PbXe collisions at $\sqrt{s_{N N}}=72 \mathrm{GeV}$ with $\int \mathcal{L}_{\mathrm{PbXe}}=30 \mathrm{nb}^{-1}$, assuming $R_{A A}=1$

Table 4 Drell-Yan yields and ratios of the yields over the combinatorial background for PbXe collisions at $\sqrt{s_{N N}}=72 \mathrm{GeV}$ and for $\mathcal{L}_{\mathrm{PbXe}}=30 \mathrm{nb}^{-1}$, in 4 di-muon invariant mass ranges between 4 and $8 \mathrm{GeV} / c^{2}$ and 3 rapidity ranges between 2 and 5 , with a single muon cut of $p_{T}^{\mu}>1.2 \mathrm{GeV} / c$ on both muons

\begin{tabular}{|c|c|c|c|c|}
\hline \multirow[t]{2}{*}{ Yields } & \multicolumn{2}{|c|}{$M_{\mu^{+} \mu^{-}}: 4-5 \mathrm{GeV} / c^{2}$} & \multicolumn{2}{|c|}{$M_{\mu^{+} \mu^{-}}: 5-6 \mathrm{GeV} / c^{2}$} \\
\hline & Signal $\left(\times 10^{3}\right)$ & $S / B\left(\times 10^{-3}\right)$ & Signal $\left(\times 10^{3}\right)$ & $S / B\left(\times 10^{-3}\right)$ \\
\hline$y: 2-3$ & 11.5 & 2.6 & 4.8 & 6.3 \\
\hline$y: 3-4$ & 26.9 & 0.2 & 15.6 & 0.4 \\
\hline \multirow[t]{3}{*}{$y: 4-5$} & 15.3 & 0.3 & 8.3 & 1.0 \\
\hline & \multicolumn{2}{|c|}{$M_{\mu^{+} \mu^{-}}: 6-7 \mathrm{GeV} / c^{2}$} & \multicolumn{2}{|c|}{$M_{\mu^{+} \mu^{-}}: 7-8 \mathrm{GeV} / c^{2}$} \\
\hline & Signal $\left(\times 10^{3}\right)$ & $S / B\left(\times 10^{-3}\right)$ & Signal $\left(\times 10^{3}\right)$ & $S / B\left(\times 10^{-3}\right)$ \\
\hline$y: 2-3$ & 1.9 & 13.9 & 0.8 & 28.1 \\
\hline$y: 3-4$ & 8.8 & 0.9 & 5.1 & 2.1 \\
\hline$y: 4-5$ & 5.1 & 3.0 & 2.8 & 6.3 \\
\hline
\end{tabular}




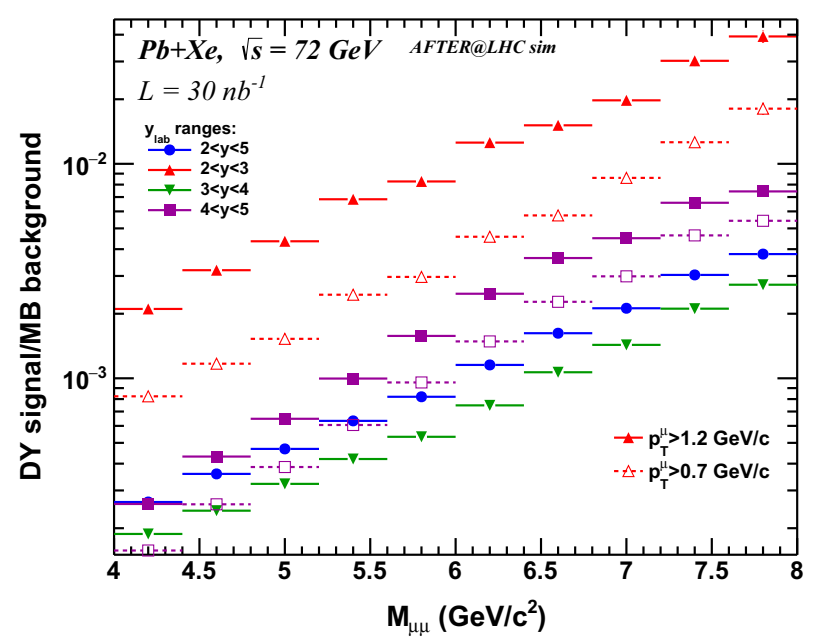

Fig. 6 Drell-Yan signal over the combinatorial background ratio as a function of di-muon invariant mass, for $2<y_{\text {lab }}<5,2$ $<y_{\text {lab }}<3,3<y_{\text {lab }}<4$ and $4<y_{\text {lab }}<5$. Solid lines represent the $S / B$ with the default Drell-Yan single muon cut of $p_{T}^{\mu}>$ $1.2 \mathrm{GeV} / c$, and dashed lines represent the $S / B$ with $p_{T}^{\mu}>0.7 \mathrm{GeV} / c$. No nuclear modifications assumed. PbXe collisions at $\sqrt{s_{N N}}=72 \mathrm{GeV}$ with $\int \mathcal{L}_{\mathrm{PbXe}}=30 \mathrm{nb}^{-1}$

measures hadrons, electrons, and photons and in a forward muon spectrometer [81,82]. The ALICE upgrade is planned after the Long Shutdown 2 (LS2) of 2018 [83] and is designed for recording data with a rate of $200 \mathrm{kHz}$ in $p p$ and $p A$ collisions and $50 \mathrm{kHz}$ in $\mathrm{PbPb}$ collisions.

The central barrel covers the pseudorapidity range $|\eta|<0.9$ around mid-rapidity, while the muon spectrometer covers the forward pseudorapidity range $2.5<\eta<4$. It should be noticed that, due to the absorber system the only available region for the installation of a fixed target in ALICE is the A-side, opposite to the muon spectrometer one.

\subsubsection{Muon Spectrometer}

In a fixed-target mode, the acceptance of the muon spectrometer allows for measurements in the rapidity regions of $-2.3<y_{\text {c.m.s. }}<-0.8$ with a $7 \mathrm{TeV}$ proton beam and of $-1.7<y_{\text {c.m.s. }}<-0.2$ with a $2.76 \mathrm{~A}$. TeV $\mathrm{Pb}$ beam, when considering the target at the nominal interaction point of the experiment.

The vertex can in principle be displaced upstream (in the A-side of ALICE) from the nominal interaction point by up to a few meters. The geometrical acceptance of the muon spectrometer as a function of the longitudinal position of the fixed target, is shown in Fig. 7 separately for the tracking system of the muon spectrometer (identified as MUON, corresponding to the tracking stations installed after the hadron absorber) and the MFT (Muon Forward Tracker) which will serve as vertex tracker for the muon spectrometer after LS2 [84].

As one can notice from Fig. 7, the geometrical acceptances of the spectrometer and the MFT decouple very early as the target is moved away from the nominal interaction point. For this reason, an optimised innertracking system would be needed, providing both vertexing capabilities and the proxy for the tracking in the spectrometer.

On the other hand, the geometrical acceptance of the spectrometer stays reasonably close to $y_{\text {c.m.s. }} \simeq 0$, keeping a range of about one unit of rapidity even for longitudinal positions of the fixed target very far from the nominal interaction point.

It should be however noted that large displacements between the fixed-target position and the hadron absorber would automatically result in an increase of the combinatorial background from semi-muonic decays of pions and kaons. This aspect should carefully be accounted for when evaluating the physics performance of the muon spectrometer in a fixed-target configuration with a displaced vertex.

\subsubsection{Central Barrel}

If the target is located at the nominal interaction point of the experiment, the central barrel covers the far backward region with $y_{\text {c.m.s. }}<-3.9$ and $y_{\text {c.m.s. }}<-3.3$ with a $7 \mathrm{TeV}$ proton beam and a $2.76 \mathrm{~A} . \mathrm{TeV} \mathrm{Pb}$ beam, 


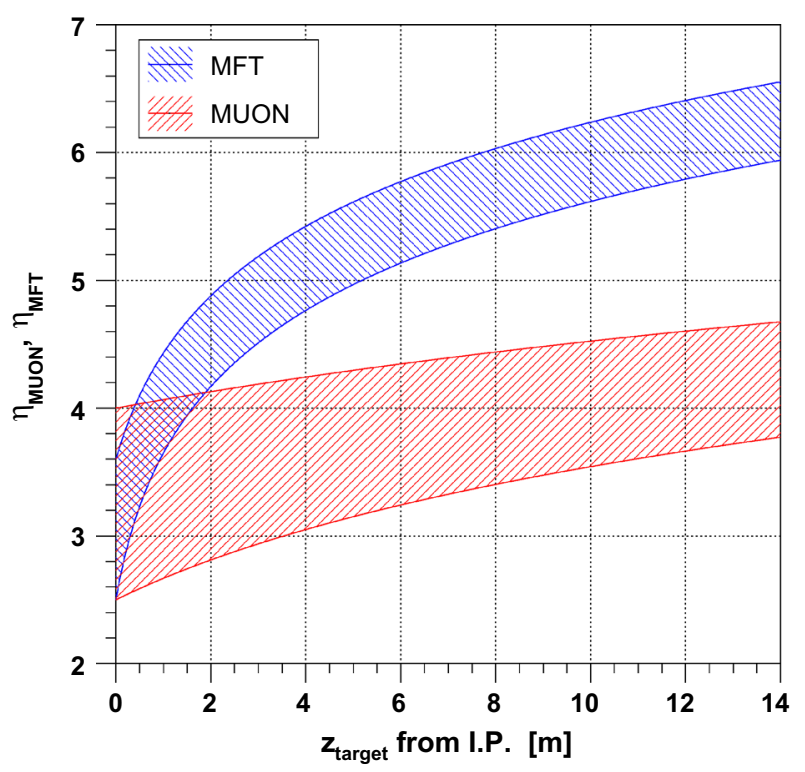

Fig. 7 Pseudorapidity ranges in the laboratory frame for the tracking chambers of the muon spectrometer (MUON) and the disks of the Muon Forward Tracker (MFT) as a function of the longitudinal position of the fixed target from the nominal interaction region

respectively. The detection and the identification of particles with the central barrel therefore seems very appealing to access far negative $x_{F}$, down to the target fragmentation region. Several questions however need to be addressed in the case of a target far from the nominal interaction point. The tracking performance of the Time Projection Chamber, Time Of Flight and Transition Radiation Detector need to be evaluated for particles entering and traversing their volume with an angular direction different from the nominal specifications.

As in the muon spectrometer case, a new vertex detector would be needed if the current ALICE Inner Tracking System (ITS) falls outside the geometrical acceptance of interest for particle production in a fixedtarget configuration. It would provide both vertexing capabilities and the proxy for the tracking in the main detectors of the central barrel.

\section{Conclusions}

We have outlined the broad spectrum of possibilities offered by AFTER@LHC for studies of the nuclear matter properties in $p A$ and heavy-ion collisions in an energy range where a phase transition to a partonic matter in heavy-ion collisions is expected.

The heavy-ion studies with the LHC beams used in the fixed-target mode include:

- the correlation of the medium temperature and the quarkonium suppression as a function of their binding energies by looking for signs of a sequential suppression with as many states as possible;

- the precise and complete determination of the Fourier expansion of identified particle azimuthal asymmetries as a function of rapidity, down to the target fragmentation region;

- the determination of different mixtures of quark-gluon plasma and cold nuclear matter effects on the rapidity- and transverse-momentum-dependent particle-yield modifications, in particular heavy-flavoured particles;

- the verification/falsification of the factorisation of the initial-state nuclear effects on Drell-Yan-pair measurements on different systems including asymmetric nucleus-nucleus collisions;

- the search for net baryon singularities from a possible QCD critical point using the beam-energy-ramp phase;

- the search for modifications in the mass width and branching ratios between hadronic and leptonic channels of $\phi, \omega$ and $\rho$ decays as a signature of the chiral-symmetry restoration in the same spirit of the NA60 studies.

As we have shown for a LHCb-like detector, various charmonium and bottomonium states can be measured with an unprecedented accuracy over wide $p_{T}$ and rapidity ranges both in $p A$ and $A A$ collisions. These studies 
will be accompanied by open heavy-flavour, light-flavour, and Drell-Yan measurements to deliver in-depth characteristics of the partonic matter and the phase transition. Based on our first acceptance studies, the ALICE detectors used in the fixed-target mode can provide complementary measurements in this new energy domain.

Acknowledgements This research was supported in part by the French P2IO Excellence Laboratory and the French CNRS via the grant FCPPL-Quarkonium4AFTER, and Ministerio de Ciencia e Innovacion of Spain and Xunta de Galicia.

Open Access This article is distributed under the terms of the Creative Commons Attribution 4.0 International License (http:// creativecommons.org/licenses/by/4.0/), which permits unrestricted use, distribution, and reproduction in any medium, provided you give appropriate credit to the original author(s) and the source, provide a link to the Creative Commons license, and indicate if changes were made.

\section{References}

1. E598 Collaboration, J.J. Aubert et al., Experimental observation of a heavy particle. J. Phys. Rev. Lett. 33, 1404-1406 (1974)

2. S.W. Herb et al., Observation of a dimuon resonance at $9.5-\mathrm{GeV}$ in $400-\mathrm{GeV}$ proton-nucleus collisions. Phys. Rev. Lett. 39, 252-255 (1977)

3. T.A. Armstrong et al., Observation of the p wave singlet state of charmonium. Phys. Rev. Lett. 69, 2337-2340 (1992)

4. NA50 Collaboration, M.C. Abreu et al., Evidence for deconfinement of quarks and gluons from the J/psi suppression pattern measured in $\mathrm{Pb}+\mathrm{Pb}$ collisions at the CERN SPS. Phys. Lett. B477, 28-36 (2000)

5. P. Hoyer, M. Vanttinen, U. Sukhatme, Violation of factorization in charm hadroproduction. Phys. Lett. B 246, 217-220 (1990)

6. NA3 Collaboration, J. Badier et al., Evidence for $\psi \psi$ production in $\pi^{-}$interactions at $150-\mathrm{GeV} / c$ and $280-\mathrm{GeV} / c$. Phys. Lett. B114, 457-460 (1982)

7. NA51 Collaboration, A. Baldit et al., Study of the isospin symmetry breaking the in the light quark sea of the nucleon from the Drell-Yan process. Phys. Lett. B332, 244-250 (1994)

8. NuSea Collaboration, E.A. Hawker et al., Measurement of the light anti-quark flavor asymmetry in the nucleon sea. Phys. Rev. Lett. 80, 3715-3718 (1998). arXiv:hep-ex/9803011 [hep-ex]

9. NuSea Collaboration, M.A. Vasilev et al., Parton energy loss limits and shadowing in Drell-Yan dimuon production. Phys. Rev. Lett. 83, 2304-2307 (1999). arXiv:hep-ex/9906010 [hep-ex]

10. NuSea Collaboration, L.Y. Zhu et al., Measurement of angular distributions of Drell-Yan Dimuons in $p+d$ interaction at 800-GeV/c. Phys. Rev. Lett. 99, 082301 (2007). arXiv:hep-ex/0609005 [hep-ex]

11. S.J. Brodsky, F. Fleuret, C. Hadjidakis, J.P. Lansberg, Physics opportunities of a fixed-target experiment using the LHC beams. Phys. Rep. 522, 239-255 (2013). arXiv:1202.6585 [hep-ph]

12. S. Koshkarev, S. Groote, Double quarkonium production at high Feynman- $x$. Nucl. Phys. B 915, 384-391 (2017). arXiv:1611.08149 [hep-ph]

13. A. Signori, Flavor and evolution effects in TMD phenomenology. PhD thesis, Vrije U., Amsterdam, 2016. http://inspirehep. net/record/1493030/files/Thesis-2016-Signori.pdf

14. S. Koshkarev, Production of the doubly heavy baryons, $B_{c}$ meson and the all-charm Tetraquark at AFTER @LHC with double intrinsic heavy mechanism. Acta Phys. Polon. B, 48(2), 163-169 (2017)

15. J.P. Lansberg et al., Single-transverse-spin-asymmetry studies with a fixed-target experiment using the LHC beams (AFTER@LHC). PoS DIS2016, 241 (2016). arXiv:1610.05228 [hep-ex]

16. J.-P. Lansberg et al., Physics case for a polarised target for AFTER@LHC. PoS PSTP2015, 042 (2016). arXiv:1602.06857 [nucl-ex]

17. A. Signori, Gluon TMDs in quarkonium production. Few Body Syst. 57(8), 651-655 (2016). arXiv:1602.03405 [hep-ph]

18. C. Pisano, Momentum imbalance observables as a probe of Gluon TMDs. PoS QCDEV2015, 024 (2015) . arXiv:1512.08143 [hep-ph]

19. R. Vogt, Gluon Shadowing effects on $J / \psi$ and $\Upsilon$ production in $\mathrm{p}+\mathrm{Pb}$ collisions at $\sqrt{s_{N N}}=115 \mathrm{GeV}$ and $\mathrm{p}+\mathrm{Pb}$ Collisions at $\sqrt{s_{N N}}=72 \mathrm{GeV}$ at AFTER $@$ LHC. Adv. High Energy Phys. 2015, 492302 (2015). arXiv:1510.03976 [hep-ph]

20. Y. Feng, J.-X. Wang, Next-to-leading order differential cross sections for, and production in proton-proton collisions at a fixed-target experiment using the LHC beams. Adv. High Energy Phys. 2015, 726393 (2015). arXiv:1510.05277 [hep-ph]

21. C. Barschel, P. Lenisa, A. Nass, E. Steffens, A gas target internal to the LHC for the study of pp single-spin asymmetries and heavy ion collisions. Adv. High Energy Phys. 2015, 463141 (2015)

22. D. Kikola, Prospects for open heavy flavor measurements in heavy ion and $p+A$ collisions in a fixed-target experiment at the LHC. Adv. High Energy Phys. 2015, 783134 (2015)

23. A.B. Kurepin, N.S. Topilskaya, Quarkonium production and proposal of the new experiments on fixed target at the LHC. Adv. High Energy Phys. 2015, 760840 (2015)

24. K. Zhou, Z. Chen, P. Zhuang, Antishadowing effect on charmonium production at a fixed-target experiment using LHC beams. Adv. High Energy Phys. 2015, 439689 (2015). arXiv:1507.05413 [nucl-th]

25. F. Arleo, S. Peigne, Quarkonium suppression from coherent energy loss in fixed-target experiments using LHC beams. Adv. High Energy Phys. 2015, 961951 (2015). arXiv:1504.07428 [hep-ph]

26. J.-P. Lansberg, H.-S. Shao, Double-quarkonium production at a fixed-target experiment at the LHC (AFTER@LHC). Nucl. Phys. B 900, 273-294 (2015). arXiv:1504.06531 [hep-ph]

27. S.J. Brodsky, A. Kusina, F. Lyonnet, I. Schienbein, H. Spiesberger, R. Vogt, A review of the intrinsic heavy quark content of the nucleon. Adv. High Energy Phys. 2015, 231547 (2015). arXiv:1504.06287 [hep-ph] 
28. L. Massacrier, B. Trzeciak, F. Fleuret, C. Hadjidakis, D. Kikola, J.P. Lansberg, H.S. Shao, Feasibility studies for quarkonium production at a fixed-target experiment using the LHC proton and lead beams (AFTER@LHC). Adv. High Energy Phys. 2015, 986348 (2015). arXiv:1504.05145 [hep-ex]

29. M. Anselmino, U. D'Alesio, S. Melis, Transverse single-spin asymmetries in proton-proton collisions at the AFTER@LHC experiment in a TMD factorisation scheme. Adv. High Energy Phys. 2015, 475040 (2015). arXiv:1504.03791 [hep-ph]

30. J.P. Lansberg, L. Szymanowski, J. Wagner, Lepton-pair production in ultraperipheral collisions at AFTER @LHC. JHEP 09, 087 (2015). arXiv:1504.02733 [hep-ph]

31. F.A. Ceccopieri, Studies of backward particle production with a fixed-target experiment using the LHC beams. Adv. High Energy Phys. 2015, 652062 (2015). arXiv:1503.05813 [hep-ph]

32. V.P. Goncalves, W.K. Sauter, $\eta_{c}$ production in photon-induced interactions at a fixed target experiment at LHC as a probe of the odderon. Phys. Rev. D 91(9), 094014 (2015). arXiv:1503.05112 [hep-ph]

33. K. Kanazawa, Y. Koike, A. Metz, D. Pitonyak, Transverse single-spin asymmetries in proton-proton collisions at the AFTER@LHC experiment. Adv. High Energy Phys. 2015, 257934 (2015). arXiv:1502.04021 [hep-ph]

34. J.P. Lansberg, Back-to-back isolated photon-quarkonium production at the LHC and the transverse-momentum-dependent distributions of the gluons in the proton. Int. J. Mod. Phys. Conf. Ser. 40, 1660015 (2016). arXiv:1502.02263 [hep-ph]

35. L. Massacrier et al., Studies of transverse-momentum-dependent distributions with a fixed-target ExpeRiment using the LHC beams (AFTER@LHC). Int. J. Mod. Phys. Conf. Ser. 40(01),1660107 (2016). arXiv:1502.00984 [nucl-ex]

36. J.P. Lansberg et al., Spin physics and TMD studies at a fixed-target expeRiment at the LHC (AFTER@LHC). EPJ Web Conf. 85, 02038 (2015). arXiv:1410.1962 [hep-ex]

37. G. Chen, X.-G. Wu, J.-W. Zhang, H.-Y. Han, H.-B. Fu, Hadronic production of $\Xi_{c c}$ at a fixed-target experiment at the LHC. Phys. Rev. D 89(7), 074020 (2014). arXiv:1401.6269 [hep-ph]

38. A. Rakotozafindrabe et al., Spin physics at a fixed-target experiment at the LHC (AFTER@LHC). Phys. Part. Nucl. 45, 336-337 (2014). arXiv:1301.5739 [hep-ex]

39. J.P. Lansberg et al., AFTER@LHC: a precision machine to study the interface between particle and nuclear physics. EPJ Web Conf. 66, 11023 (2014). arXiv:1308.5806 [hep-ex]

40. J.P. Lansberg et al. Prospects for a fixed-target experiment at the LHC: AFTER@LHC. PoS ICHEP2012, 547 (2013). arXiv: 1212.3450 [hep-ex]

41. C. Lorce et al., Spin and diffractive physics with a fixed-target experiment at the LHC (AFTER@LHC). AIP Conf. Proc. 1523,149 (2012). arXiv:1212.0425 [hep-ex]

42. A. Rakotozafindrabe et al., Ultra-relativistic heavy-ion physics with AFTER@LHC. Nucl. Phys. A904-905, 957c-960c (2013). arXiv:1211.1294 [nucl-ex]

43. D. Boer, C. Pisano, Polarized gluon studies with charmonium and bottomonium at LHCb and AFTER. Phys. Rev. D 86, 094007 (2012). arXiv:1208.3642 [hep-ph]

44. J.P. Lansberg et al., A fixed-target experiment at the LHC (AFTER@LHC) : luminosities, target polarisation and a selection of physics studies. PoS QNP2012, 049 (2012). arXiv:1207.3507 [hep-ex]

45. J.P. Lansberg, S.J. Brodsky, F. Fleuret, C. Hadjidakis, Quarkonium physics at a fixed-target experiment using the LHC beams. Few Body Syst. 53, 11-25 (2012). arXiv:1204.5793 [hep-ph]

46. T. Liu, B.-Q. Ma, Azimuthal asymmetries in lepton-pair production at a fixed-target experiment using the LHC beams (AFTER). Eur. Phys. J. C 72, 2037 (2012). arXiv:1203.5579 [hep-ph]

47. D. Kikola, M.G. Echevarria, C. Hadjidakis, J.-P. Lansberg, C. Lorce, L. Massacrier, C.M. Quintans, A. Signori, and B. Trzeciak, Feasibility Studies for single transverse-spin asymmetry measurements at a fixed-target experiment using the LHC proton and lead beams (AFTER@LHC). Few Body Syst. 58(4), 139 (2017). arXiv:1702.01546 [hep-ex]

48. A. Andronic et al., Heavy-flavour and quarkonium production in the LHC era: from protonproton to heavy-ion collisions. Eur. Phys. J. C76(3) 107, (2016). arXiv:1506.03981 [nucl-ex]

49. N. Brambilla et al., Heavy quarkonium: progress, puzzles, and opportunities. Eur. Phys. J. C 71, 1534 (2011). arXiv:1010.5827 [hep-ph]

50. J.P. Lansberg, $J / \psi, \psi^{\prime}$ ' and $\Upsilon$ production at hadron colliders: a review. Int. J. Mod. Phys. A 21, 3857-3916 (2006). arXiv:hep-ph/0602091

51. A. Andronic, P. Braun-Munzinger, K. Redlich, J. Stachel, Statistical hadronization of charm in heavy ion collisions at SPS, RHIC and LHC. Phys. Lett. B571, 36-44 (2003). arXiv:nucl-th/0303036 [nucl-th]

52. T. Matsui, H. Satz, J/ $\psi$ suppression by Quark-Gluon plasma formation. Phys. Lett. B 178, 416-422 (1986)

53. P. Braun-Munzinger, V. Koch, T. Schfer, J. Stachel, Properties of hot and dense matter from relativistic heavy ion collisions. Phys. Rep. 621, 76-126 (2016). arXiv:1510.00442 [nucl-th]

54. L. Grandchamp, R. Rapp, Charmonium suppression and regeneration from SPS to RHIC. Nucl. Phys. A709, 415-439 (2002). arXiv:hep-ph/0205305 [hep-ph]

55. X. Du, R. Rapp, Sequential regeneration of charmonia in heavy-ion collisions. Nucl. Phys. A 943, 147-158 (2015). arXiv: 1504.00670 [hep-ph]

56. NA50 Collaboration, M.C. Abreu et al., Anomalous J/psi suppression in $\mathrm{Pb}-\mathrm{Pb}$ interactions at $158 \mathrm{GeV} / \mathrm{c}$ per nucleon. Phys. Lett. B410, 337-343 (1997)

57. P. Faccioli, J. Seixas, Observation of $\chi_{c}$ and $\chi_{b}$ nuclear suppression via dilepton polarization measurements. Phys. Rev. D 85, 074005 (2012). arXiv:1203.2033 [hep-ph]

58. P. Faccioli, C. Lourenco, J. Seixas, H.K. Wohri, J/psi polarization from fixed-target to collider energies. Phys. Rev. Lett. 102, 151802 (2009). arXiv:0902.4462 [hep-ph]

59. P. Faccioli, V. Knnz, C. Lourenco, J. Seixas, H.K. Whri, Quarkonium production in the LHC era: a polarized perspective. Phys. Lett. B 736, 98-109 (2014). arXiv:1403.3970 [hep-ph]

60. H. Satz, Colour deconfinement and quarkonium binding. J. Phys. G32, R25 (2006). arXiv:hep-ph/0512217 [hep-ph]

61. STAR Collaboration, L. Adamczyk et al., Observation of $D^{0}$ meson nuclear modifications in Au + Au collisions at $\sqrt{s_{N N}}=$ 200 GeV. Phys. Rev. Lett.113(14), 142301 (2014). arXiv:1404.6185 [nucl-ex] 
62. PHENIX Collaboration, A. Adare et al., Energy loss and flow of heavy auarks in $\mathrm{Au}+\mathrm{Au}$ collisions at $\mathrm{s}(\mathrm{NN}) * *(1 / 2)=$ 200-GeV. Phys. Rev. Lett. 98, 172301 (2007). arXiv:nucl-ex/0611018 [nucl-ex]

63. J.-Y. Ollitrault, Anisotropy as a signature of transverse collective flow. Phys. Rev. D 46, 229-245 (1992)

64. P.F. Kolb, J. Sollfrank, U.W. Heinz, Anisotropic transverse flow and the quark hadron phase transition. Phys. Rev. C 62, 054909 (2000). arXiv:hep-ph/0006129 [hep-ph]

65. P. Naselsky et al., Morphology of high-multiplicity events in heavy ion collisions. Phys. Rev. C 86, 024916 (2012). arXiv: 1204.0387 [hep-ph]

66. I.A. Karpenko, P. Huovinen, H. Petersen, M. Bleicher, Estimation of the shear viscosity at finite net-baryon density from $A+A$ collision data at $\sqrt{s_{\mathrm{NN}}}=7.7-200 \mathrm{GeV}$. Phys. Rev. C91(6), 064901 (2015). arXiv:1502.01978 [nucl-th]

67. NA60 Collaboration, R. Arnaldi et al., First measurement of the rho spectral function in high-energy nuclear collisions. Phys. Rev. Lett. 96, 162302 (2006). arXiv:nucl-ex/0605007 [nucl-ex]

68. B. Borasoy, Introduction to chiral perturbation theory. Springer Proc. Phys. 118, 1-26 (2008). arXiv:hep-ph/0703297 [hep-ph]

69. F. Karsch, E. Laermann, Susceptibilities, the specific heat and a cumulant in two flavor QCD. Phys. Rev. D50, 6954-6962 (1994). arXiv:hep-lat/9406008 [hep-lat]

70. H.-S. Shao, HELAC-Onia: an automatic matrix element generator for heavy quarkonium physics. Comput. Phys. Commun. 184, 2562-2570 (2013). arXiv:1212.5293 [hep-ph]

71. H.-S. Shao, HELAC-Onia 2.0: an upgraded matrix-element and event generator for heavy quarkonium physics. Comput. Phys. Commun. 198, 238-259 (2016). arXiv:1507.03435 [hep-ph]

72. J. Alwall et al., A Standard format for Les Houches event files. Comput. Phys. Commun. 176, 300-304 (2007). arXiv:hep-ph/0609017 [hep-ph]

73. T. Sjostrand, S. Mrenna, P.Z. Skands, A brief introduction to PYTHIA 8.1. Comput. Phys. Commun. 178, $852-867$ (2008). arXiv:0710.3820 [hep-ph]

74. Particle Data Group Collaboration, J. Beringer et al., Review of particle physics (RPP). Phys. Rev. D86, 10001 (2012)

75. A. Buckley, J. Ferrando, S. Lloyd, K. Nordstrm, B. Page, M. Rfenacht, M. Schnherr, G. Watt, LHAPDF6: parton density access in the LHC precision era. Eur. Phys. J. C 75, 132 (2015). arXiv:1412.7420 [hep-ph]

76. LHCb Collaboration, A.A. Alves, Jr. et al., The LHCb detector at the LHC. JINST 3, S08005 (2008)

77. F. Archilli et al., Performance of the muon identification at LHCb. JINST 8, P10020 (2013). arXiv:1306.0249 [physics.ins-det]

78. LHCb Collaboration, R. Aaij et al., LHCb detector performance. Int. J. Mod. Phys. A30(07), 1530022 (2015). arXiv:1412.6352 [hep-ex]

79. B. Alver, M. Baker, C. Loizides, and P. Steinberg, The PHOBOS Glauber Monte Carlo. arXiv:0805.4411 [nucl-ex]

80. C. Loizides, J. Nagle, P. Steinberg, Improved version of the PHOBOS Glauber Monte Carlo. SoftwareX 1-2, 13-18 (2015). arXiv:1408.2549 [nucl-ex]

81. ALICE Collaboration, K. Aamodt et al., The ALICE experiment at the CERN LHC. JINST 3, S08002 (2008)

82. ALICE Collaboration, B.B. Abelev et al., Performance of the ALICE experiment at the CERN LHC. Int. J. Mod. Phys. A29,1430044, (2014). arXiv:1402.4476 [nucl-ex]

83. ALICE Collaboration, B. Abelev et al., Upgrade of the ALICE experiment: letter of intent. J. Phys. G41, 087001 (2014)

84. Technical Design Report for the Muon Forward Tracker. Tech. Rep. CERN-LHCC-2015-001. ALICE-TDR-018, Jan, 2015, https://cds.cern.ch/record/1981898 